\title{
Laparoendoscopic single-site oophorectomy for a right ovarian borderline serous tumor of stage IC2, a literature review
}

\begin{abstract}
Background: Ovarian cancer represents approximately $30 \%$ of all female reproductive organ cancers and is the leading cause of death for gynecological cancer in the United States. Borderline ovarian tumors comprise approximately $15-20 \%$ of all epithelial ovarian tumors and within that group, serous borderline ovarian tumors comprise $53 \%$. Serous borderline tumors (SBT) display atypical epithelial proliferation of serous type cells, outnumbering benign epithelial cells but have a low malignancy potential (borderline). By definition SBT has no gross stromal invasion but it can be subcategorized to those with or without microinvasion. Carcinomas make up $90 \%$ of ovarian tumors and differentiating between the distinct types of tumors via unique features is important for treatment.
\end{abstract}

Case: The patient was a 29-year-old G0P0, who presented to our clinic for evaluation of intermenstrual bleeding. Upon physical exam, a palpable, $5 \mathrm{~cm} \mathrm{x} 4 \mathrm{~cm}$ non-tender, mobile mass in the right adnexa was noted. Pelvic ultrasound revealed a $4.9 \mathrm{~cm} \times 3.7 \mathrm{~cm}$ hypoechoic mass in the right adnexa. The mass was noted to be heterogeneous with smooth borders and a preliminary diagnosis of ovarian cystadenoma was made. The patient in this case presented with a stage $1 \mathrm{C} 2$ ovarian cancer and was treated with single incision laparoscopic surgery (SILS) right salpingo-oophorectomy.

Conclusion: Ovarian cancer is the leading cause of gynecological cancers in women in the US and early diagnosis/staging is important in predicting progression-free survival. Instead of laparotomy, we utilized single-incision laparoscopic surgery to drain the patient's pelvic effusion and excise the right ovary and fallopian tube, which revealed an ovarian borderline serous tumor. This technique was conservative and fertility-sparing because it left the uterus, left ovary and left fallopian tube.

Keywords: borderline serous tumor, early stage ovarian cancer, epithelial tumor, oophorectomy, ovarian cancer, ovarian cyst, ovarian epithelial tumor, ovarian tumor, single-incision laparoscopic surgery (SILS), stage I ovarian cance, serous tumor, stage IC2 ovarian cancer
Volume 9 Issue 4 - 2018

\author{
Shadi Rezai, ${ }^{1,5}$ Alexander C Hughes, ${ }^{2}$ \\ Chunyan Zeng, ${ }^{3}$ Emily Wang, ${ }^{2}$ Yuyan Li, ${ }^{3}$ Juan \\ Liu, ${ }^{3}$ Cassandra E Henderson, ${ }^{4}$ Xiaoming \\ Guan $^{5}$ \\ 'Department of Obstetrics and Gynecology, Southern California \\ Kaiser Permanente, USA \\ ${ }^{2}$ St. George's University, School of Medicine, Grenada \\ ${ }^{3}$ Key Laboratory for Major Obstetric Diseases of Guangdong \\ Province, Department of Obstetrics and Gynecology, The Third \\ Affiliated Hospital of Guangzhou Medical University, China \\ ${ }^{4}$ Maternal and Fetal Medicine, Department of Obstetrics and \\ Gynecology, Lincoln Medical and Mental Health Center, USA \\ ${ }^{5}$ Division of Minimally Invasive Gynecologic Surgery, \\ Department of Obstetrics and Gynecology, Baylor College of \\ Medicine, USA
}

\begin{abstract}
Correspondence: Juan Liu MD PhD, Department of Obstetrics and Gynecology, The Third Affiliated Hospital of Guangzhou Medical University, Guangzhou 510150, China. Tel +86-20-8I 292697, Fax +86-20-8I292697,

Email liujuan900II@I63.com

Xiaoming Guan MD PhD, Section Chief and Fellowship Director, Division of Minimally Invasive Gynecologic Surgery, Department of Obstetrics and Gynecology, Baylor College of Medicine, 665I Main Street, I0th Floor, Houston, Texas, 77030, USA, Tel (832) 826-7464, Fax (832) 825-9349, Email xiaoming@bcm.edu
\end{abstract}

Received: July 09, 2018 | Published: August 16, 2018

\section{Background}

Ovarian cancer represents approximately $30 \%$ of all female reproductive organ cancers and is the leading cause of death for gynecological cancer in the United States. ${ }^{1}$ Borderline ovarian tumors comprise approximately $15-20 \%$ of all epithelial ovarian tumors and within that group, serous borderline ovarian tumors comprise $53 \%{ }^{2,3}$ Serous borderline tumors (SBT) display atypical epithelial proliferation of serous type cells, outnumbering benign epithelial cells but have a low malignancy potential (borderline). ${ }^{4,5}$ By definition SBT has no gross stromal invasion but it can be subcategorized to those with or without microinvasion. ${ }^{4}$ Carcinomas make up $90 \%$ of ovarian tumors and differentiating between the distinct types of tumors via unique features is important for treatment.

SBT tumors are usually asymptomatic, but may present with abdominal enlargement or pelvic pain, and are related to a high rate of infertility in young women. ${ }^{1}$ The mean age of diagnosis of $1 \mathrm{C}$ ovarian tumor is 51.7 years. ${ }^{6}$ According to a long term follow up study, SBT tumors have a 5 year survival rate of $100 \%$ and a 10 year survival rate of $86 \%{ }^{1}{ }^{1}$ The cure rate for serous borderline tumors is close to $100 \%$ especially when they are diagnosed at stage $1 .^{2,7}$

Cancer staging allows for understanding of the patient's condition, prognosis, and treatment options. Staging for ovarian cancer is guided by the International Federation of Gynecology and Obstetrics (FIGO) which was last updated in $2014 .^{8}$ The patient in this case presented with a stage $1 \mathrm{C} 2$ ovarian cancer and was treated with single incision laparoscopic surgery (SILS) right salpingo-oophorectomy.

\section{Presentation of the case}

The patient was a 29-year-old Gravida 0, Para 0, who presented to our clinic for evaluation of intermenstrual bleeding. She endorsed regular menstrual cycles of 28 days with menses lasting 4-6 days and denied pelvic pain, dysmenorrhea and dyspareunia. Upon physical exam, a palpable, $5 \mathrm{~cm} \times 4 \mathrm{~cm}$ non-tender, mobile mass in the right adnexa was noted. Pelvic ultrasound revealed a $4.9 \mathrm{~cm} \times 3.7 \mathrm{~cm}$ hypoechoic mass in the right adnexa. The mass was noted to be heterogeneous with smooth borders and a preliminary diagnosis of ovarian cystadenoma was made.

Computed tomography (CT) of the pelvis (Figure 1) showed a cystic mass in the right adnexa suspicious for ovarian borderline epithelial tumor or cystadenocarcinoma. A small amount of free fluid in the cul-de-sac and endometrial thickening were also noted (Figure $1)$.

The patient underwent hysteroscopy, single incision laparoscopic surgery (SILS), right salpingo-oophorectomy (RSO), uterine surface 
nodule resection and endometrial curettage.
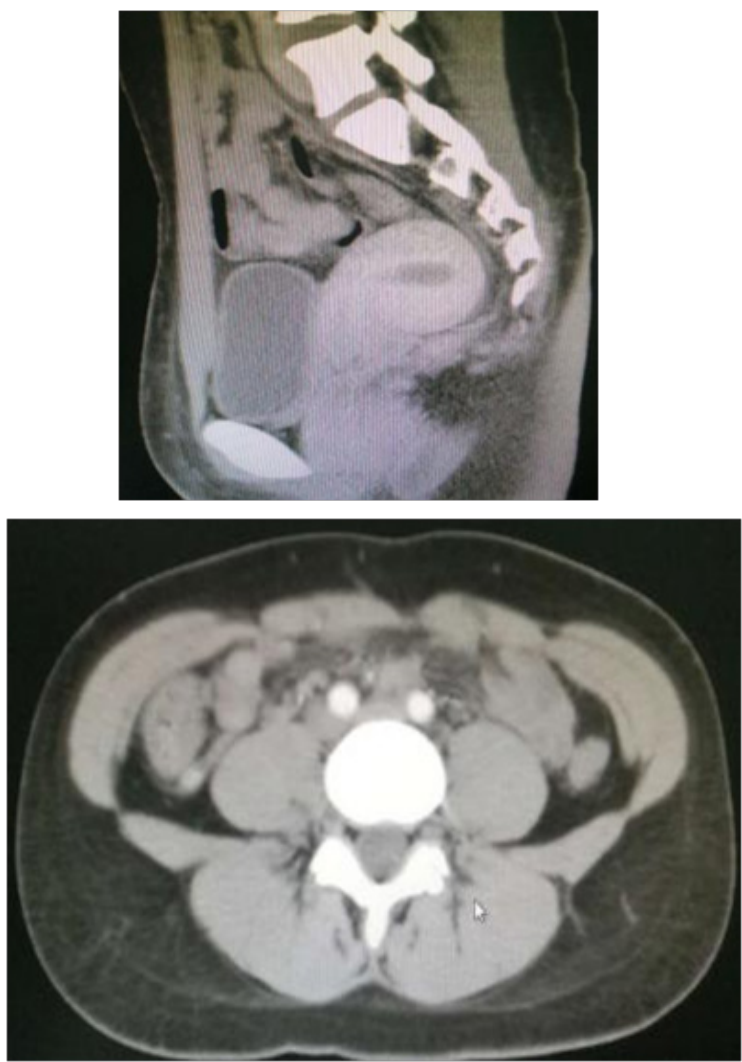

Figure I CT Scan of pelvis:The volume of the uterus is full, density of uterine wall is well-distributed, endometrium slightly thickened, enhanced scan show no lesions, solid-cystic hypodense shadow was seen in the right-sided adnexa, cystic lesion which is in the size of $4.3 \mathrm{~cm} \times 3.8 \mathrm{~cm} \times 5.3 \mathrm{~cm}$ is predominant. In the cystic lesion, we can see line like separate shadow, papillary nodules can be seen in its top wall; papillary nodules are in the size of $1.0 \mathrm{~cm} \times 0.6 \mathrm{~cm} \times 0.6 \mathrm{~cm}$. The demarcation between the lesion and the bladder or adjacent intestine was clear. No lymphadenopathy noted. ${ }^{20}$

Hysteroscopy revealed a normal cervix and a normal sized uterus. The endometrium was pale red, thick, uniform with visible glandular openings and two pink tongue pattern lesions on the posterior wall. Endometrial curettage for the clinical diagnosis of endometrial hyperplasia was performed.

Single incision laparoscopy revealed a normal sized uterus and a small $(20 \mathrm{ml})$ clear yellow pelvic effusion which was drained and sent for cytology. Four endometriosis lesions, $2 \mathrm{~mm}$ in size were observed on the posterior uterine wall and there were multiple white $3 \mathrm{~mm}$ nodules on the anterior uterus close to the round ligament. The left ovary and bilateral fallopian tubes were normal. The right ovary was observed to have surface papillary lesions, which were excised and sent for frozen pathology and revealed to be an ovarian borderline serous tumor. The right ovary and fallopian tube were removed. Omentum and bilateral lymph nodes were removed for staging. Peritoneal washings were performed and sent for cytology which was negative.

Postoperative pathology of the right ovary revealed serous borderline tumors and corpus luteum cysts. (Figure 2) (Figure 3) The right fallopian tube was negative for malignancy. An endometrial specimen showed early secretory stage but no tumor involvement. The uterine surface nodules were determined to be leiomyoma.
Omentum and bilateral lymph nodes were negative for metastasis. Therefore, with SBT limited to the surface of one ovary it was staged as $1 \mathrm{C} 2$, which is defined as a tumor that is on the outer surface of at least one of the ovaries or fallopian tubes or the capsule has ruptured before surgery. ${ }^{8}$

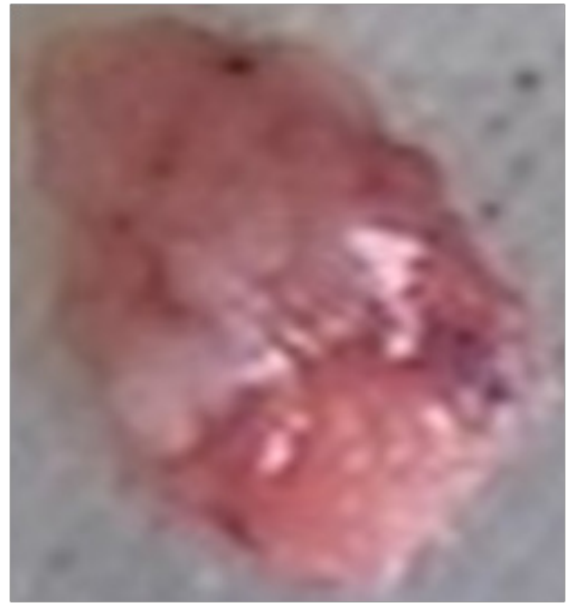

Figure 2 Pathology: Gross appearance of right ovarian tumor with papillary surface: Gray red tissue measuring $\mathrm{Icm} \times 0.7 \mathrm{~cm} \times 0.4 \mathrm{~cm}$
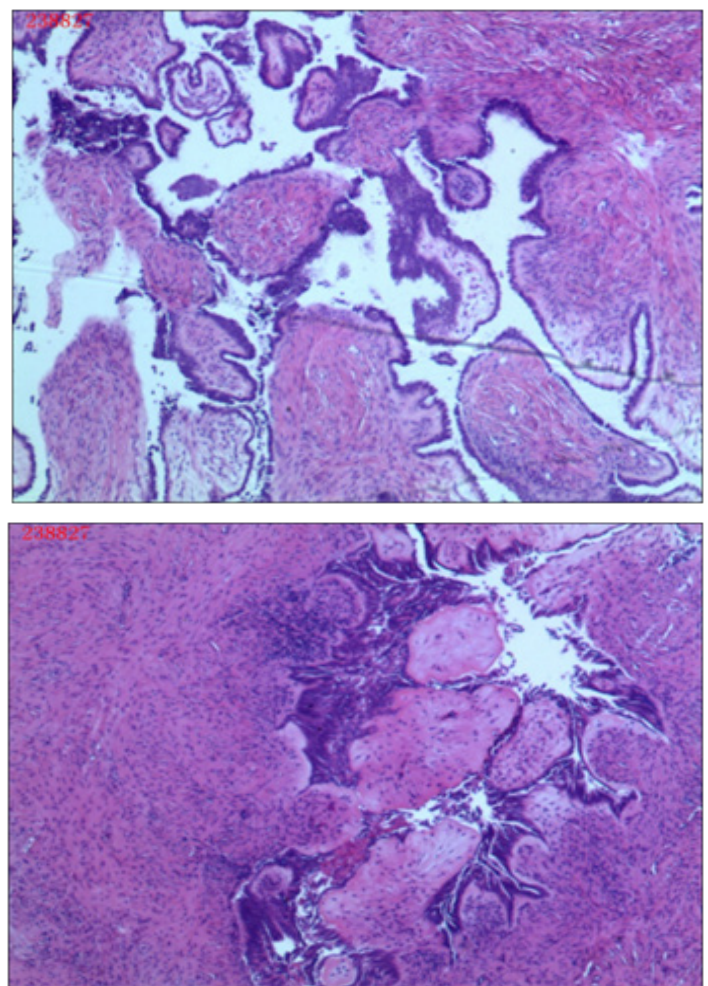

Figure 3 Pathology slide showing borderline serous tumor of right ovary.

A drainage tube was placed in the subcutaneous fat which remained after surgery. There was plenty of lymph drainage in the immediate postoperative period. By postoperative day 7 the drainage had slowed to $50 \mathrm{ml}$ and was removed. The remainder of the patient's recovery was uncomplicated and she was discharged on postoperative day 15 .

On postoperative follow up visit, 4 months after the surgery, the patient reported regular menstrual cycles with complete resolution 
of intermenstrual bleeding, but complained of slight right adnexal tenderness just before start of her menses. She is being followed and monitored by serial tumor maker (AFP, CEA, CA125, CA153, CA199, CA50 and serum ferritin) and abdominal/pelvic ultrasound, which have been normal up to this date

\section{Discussions}

The patient presented with intermenstrual bleeding and although a mass was detected on physical exam the patient did not complain of any symptoms such as pelvic pain, heaviness or dysuria. This presentation is less common for SBT as it does not have any of the symptoms outlined in the 2007 ovarian cancer consensus statement.? Of note, these recommendations have not shown to decrease mortality and were developed because ovarian disease was often found at late stages. ${ }^{7}$

Traditionally surgical staging for ovarian cancer has been done with laparotomy but advances in minimally invasive surgery have made endoscopic surgical staging more common. There has been concern of under-staging when laparotomy is not used but there is no conclusive evidence endoscopic surgery results in higher rates of recurrence or increased mortality. ${ }^{9,10}$ A study by Song et al. found multi port and single port laparoscopy to be a viable option for surgical staging of borderline ovarian tumors. ${ }^{9}$ In this case we used the SILS technique, which has shown to have improved outcomes for cosmetics and pain. ${ }^{11}$ Additionally, SILS has shown to reduce this risk of rupture especially when the ovarian cyst is large. ${ }^{9}$

Because SBT is more common than other types of GYN cancers in reproductive aged women and is associated with infertility, fertility sparing surgery (FSS) is often chosen. ${ }^{4}$ FSS, sometimes referred to as conservative surgery, involves preservation of the uterus and at least one ovary. Unfortunately, fertility after conservative treatment SBT is difficult to assess given the multivariable nature and long follow up required. Not all women attempt to conceive and some pathologies may have existed before treatment. Recently more studies have attempted to quantify the success of fertility after conservative surgery. ${ }^{12}$ This should be considered and discussed with the patient prior to treatment and may include fertility counseling.

Recently, Lee et al. compared two subtypes of fertility sparing surgery for borderline ovarian tumors and found they had a high rate of pregnancy after surgery where 79.2 and $87.5 \%$ respectively of the patients were able to conceive and most carried to term. ${ }^{13}$ Similar results were published by Song in 2011 and Park in 2009 with fertility rates upwards of $70 \%$ but in 2011 Kanat Pektas had a rate of only $52 \% .{ }^{14-16} \mathrm{~A}$ meta analysis by Vasconcelos in 2015 revealed an overall rate of approximately $50-60 \%$ and was consistent with a previous meta analysis done by Darais in 2011. ${ }^{17,18}$ Even though published results vary, it is important to note when considering conservative surgery that many studies have show it does not increase mortality even if recurrence is higher. ${ }^{18,19}$

According to a retrospective review of borderline ovarian tumors (BOT) from 1979 to 2008 consisting of 266 patients, only $8.6 \%$ of the entire cohort developed recurrent disease.$^{20}$ In another retrospective study of borderline ovarian tumors from 1984 to 2008 involving of 233 patients, 21 patients developed recurrent disease. ${ }^{21}$ There are multiple prognostic factors that influence recurrence rates in patients with borderline ovarian tumors, such as age at diagnosis, baseline CA125, stage, and histology. Progression-free survival (PFS), defined as the time of diagnosis to the time of recurrence, death or last follow up, is used to assess these prognostic factors. Those with an elevated baseline of CA-125 had a $87 \%$ PFS at 3 years and those with a normal baseline of CA- 125 had a $97 \%$ PFS at 3 years. ${ }^{20,22,23}$ Another important prognostic factor for serous BOTs is the presence of micropapillary patterns, which has a 3 year PFS rate of $76 \%$, compared to the absence of micropapillary patterns, which has a 3 year PFS of $94 \% .^{20,24-26}$

\section{Conclusion}

Ovarian cancer is the leading cause of gynecological cancers in women in the US and early diagnosis/staging is important in predicting progression-free survival. Instead of laparotomy, we utilized single-incision laparoscopic surgery to drain the patient's pelvic effusion and excise the right ovary and fallopian tube, which revealed an ovarian borderline serous tumor. This technique was conservative and fertility-sparing because it left the uterus, left ovary and left fallopian tube. Even though mixed results have been recorded on the rate of fertility after conservative surgery there is conclusive evidence to suggest that conservative surgery does not effect the mortality rate of patients with SBT. ${ }^{15}$ Even if recurrence does occur frequently with FSS they are easily treated again. Therefore with no effect on mortality, conservative surgery allows for the opportunity of conceiving after SBT.

\section{Acknowledgments}

Dr. Xiaoming Guan is a speaker for Applied Medical, Rancho Santa Margarita, and California.

\section{Conflicts of interest}

Authors did not report any potential conflicts of interests.

\section{References}

1. Kurman RJ, Carcangiu ML, Herrington CS, et al. WHO Classification of Tumors of Female Reproductive Organs. 4th ed. IARC; 2014. 307 p.

2. Morris RT, Gershenson DM, Silva EG, et al. Outcome and reproductive function after conservative surgery for borderline ovarian tumors. Obstet Gynecol. 2000;95(4):541-7.

3. Fischerova D, Zikan M, Dundr P, et al. Diagnosis, treatment, and followup of borderline ovarian tumors. Oncologist. 2012;17(12):1515-33.

4. Cadron I, Leunen K, Van Gorp T, et al. Management of borderline ovarian neoplasms. J Clin Oncol. 2007;25(20):2928-37.

5. BohîlȚea RE, Bacalbaşa N, Țurcan N, et al. Bilateral serous surface papillary borderline ovarian tumor in 19-year-old patient. Ultrasound, immunohistochemical and therapeutic particularities of reproductive age. Rom J Morphol Embryol. 2017;58(3):989-995.

6. Suh DH, Kim TH, Kim JW, et al. Improvements to the FIGO staging for ovarian cancer: reconsideration of lymphatic spread and intraoperative tumor rupture. J Gynecol Oncol. 2013;24(4):352-8.

7. Levine DA, De Los Santos JF, Fleming GF, et al. Handbook for Principles and Practice of Gynecologic Oncology. Lippincott Williams \& Wilkins; 2010. 288 p.

8. Mutch DG, Prat J. 2014 FIGO staging for ovarian, fallopian tube and peritoneal cancer. Gynecol Oncol. 2014;133(3):401-4.

9. Song T, Kim MK, Jung YW, et al. Minimally invasive compared with open surgery in patients with borderline ovarian tumors. Gynecol Oncol. 2017;145(3):508-512. 
10. Jiao X, Hu J, Zhu L. Prognostic Factors for Recurrence After FertilityPreserving Surgery in Patients With Borderline Ovarian Tumors: A Systematic Review and Meta-analysis of Observational Studies. Int $J$ Gynecol Cancer. 2017;27(9):1833-1841.

11. Fader AN, Levinson KL, Gunderson CC, et al. Laparoendoscopic singlesite surgery in gynecology: A new frontier in minimally invasive surgery. J Minim Access Surg. 2011;7(1):71-7.

12. Yasmeen S, Hannan A, Sheikh F, et al. Borderline tumors of the ovary: A clinicopathological study. Pak J Med Sci. 2017;33(2):369-373.

13. Lee SY, Choi MC, Kwon BR, et al. Oncologic and obstetric outcomes of conservative surgery for borderline ovarian tumors in women of reproductive age. Obstet Gynecol Sci. 2017;60(3):289-295

14. Song T, Hun Choi C, Lee YY, et al. Oncologic and reproductive outcomes of cystectomy compared with oophorectomy as a treatment for borderline ovarian tumors. Hum Reprod. 2011;26(8):2008-14.

15. Kanat-Pektas M, Ozat M, Gungor T, et al. Fertility outcome after conservative surgery for borderline ovarian tumors: a single center experience. Arch Gynecol Obstet. 2011;284(5):1253-8.

16. Park JY, Kim DY, Kim JH, et al. Surgical management of borderline ovarian tumors: The role of fertility-sparing surgery. Gynecol Oncol. 2009;113(1):75-82.

17. Darai E, Fauvet R, Uzan C, et al. Fertility and borderline ovarian tumor: a systematic review of conservative management, risk of recurrence and alternative options. Hum Reprod Update. 2013;19(2):151-66.

18. Vasconcelos I, de Sousa Mendes M. Conservative surgery in ovarian borderline tumors: a meta-analysis with emphasis on recurrence risk. Eur J Cancer. 2015;51(5):620-31.
19. Qian XQ, Hua XP, Wu JH, et al. Clinical Predictors of Recurrence and Prognostic Value of Lymph Node Involvement in the Serous Borderline Ovarian Tumor. Int J Gynecol Cancer. 2018;28(2):279-284.

20. Avril S. Histopathological markers of treatment response and recurrence risk in ovarian cancers and borderline tumors. Pathologe. 2017;38(Suppl 2):180-191.

21. Shih KK, Zhou Q, Huh J, et al. Risk factors for recurrence of ovarian borderline tumors. Gynecol Oncol. 2011;120(3):480-4.

22. Malpica A, Longacre TA. Prognostic indicators in ovarian serous borderline tumours. Pathology. 2018;50(2):205-213.

23. Chen $\mathrm{X}$, Fang $\mathrm{C}$, Zhu $\mathrm{T}$, et al. Identification of factors that impact recurrence in patients with borderline ovarian tumors. J Ovarian Res. 2017;10(1):23.

24. Nakai G, Yamada T, Yamamoto K, et al. MRI appearance of ovarian serous borderline tumors of the micropapillary type compared to that of typical ovarian serous borderline tumors: radiologic-pathologic correlation. J Ovarian Res. 2018;11(1):7.

25. Wu TI, Lee CL, Wu MY, et al. Prognostic factors predicting recurrence in borderline ovarian tumors. Gynecol Oncol. 2009;114(2):237-41.

26. Nougaret S, Lakhman Y, Molinari N, et al. CT Features of Ovarian Tumors: Defining Key Differences Between Serous Borderline Tumors and Low-Grade Serous Carcinomas. AJR Am J Roentgenol. 2018;210(4):918-926. 